\title{
Poverty Data Retrieval Services Blockchain based on local governments
}

\author{
Moh. Hidayat Koniyo ${ }^{1}$, Made Sudarma ${ }^{2}$, Moh. Syafri Tuloli ${ }^{3}$ \\ ${ }^{1}$ Department of Informatics Engineering, State University of Gorontalo, Gorontalo, Indonesia \\ ${ }^{2}$ Department of Electrical Engineering, University Udayana, Bali, Indonesia \\ ${ }^{3}$ Department of Informatics Engineering, State University of Gorontalo, Gorontalo, Indonesia
}

Correspondence Author: Moh. Hidayat Koniyo, Department of Informatics Engineering, State University of Gorontalo, Gorontalo, Indonesia.

Received date: 15 August 2019, Accepted date: 30 November 2019, Online date: 31 December 2019

Copyright: (C) 2019Moh. Hidayat Koniyo et al, This is an open-access article distributed under the terms of the Creative Commons Attribution License, which permits unrestricted use, distribution, and reproduction in any medium, provided the original author and source are credited.

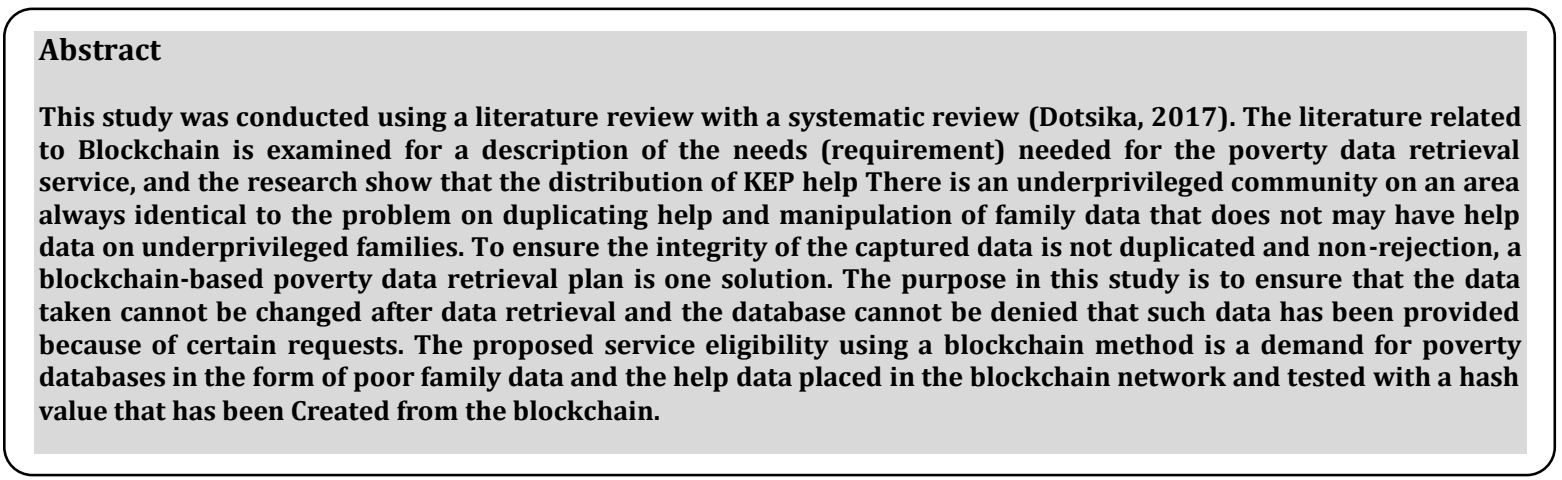

Keywords: Poverty Data, integrity, non-rejection, blockchain

\section{INTRODUCTION}

The grants provided by the Government of Gorontalo, today are classified in various types of help, with the expectation of every underprivileged family after getting help can improve the level of his life, so that society is lacking Capable each year can be suppressed in number. The distribution of aid to the underprivileged community in the local government has used a particular application to distribute aid for the impoverished community. In principle these types of help are the same and channeled to poor families can only have a kind of help, fixing and granting help has been using an application that selects the data of poor families and the provision of Help, but in implementation, there is still found a duplication of help or changes in the help to a family that is included in the category of acceptance of support. Poverty database every time there is a change in the status of poor families that are processed annually if the family has been with help is expected to improve the level of his life until it does not include the next year in the family Poor. Thus, it is important to ensure that data cannot be manipulated retrospectively and users, in this case, can have data evidence of what they fetch from the database at a specified point in time because of certain requests. Reliable data retrieval services must meet at least two requirements i.e. integrity and non-rejection (Kleinaki, 2018). Integration means that they cannot change the quern and the captured data (either accidentally or intentionally), once the retrieval operation is completed. Non-Repudiation, in this context, means recall the re-search operation in the past, poverty search services could not be legally denied that the service must have provided the data in response to the requests provided on Given time (Kleinaki, 2018). This is precisely the need for a blockchain-based poverty data retrieval service that can seal the demands and results of each given party each time the parties request help to a family with help from the Poverty database, so things related to the duplication of lowincome family data and status are inevitable.

\section{LIBRARY OVERVIEW}

\subsection{Integrity and Non-rejection}

Data integrity and renunciation are right-subject cases. The sample report (Vigil, 2015) is classified and relates different methods that continue to complete unity, truthfulness, non-renunciation and verification of presence. (Iankoulova, 2012) presents 
an extensive and structured assessment of care provisions and explanations in cloud computing. Therefore, some new exciting survey reports, in full-adjusted information processing fields, distributed in impaired (Derbeko, 2016) and the car ad hoc network (VANET) (Qu, 2015), check the surveillance and privacy features of this technology.

A method applied to protect data integrity is rearing up data, using checksum techniques or using cryptographic hash functions (Katz, 1996). The most familiar approaches use cryptographic hash functions that have high data statistics and output state-check bit arrays. This is a one-moving serve, for example, it is not relevant to measure input from production, and it is deterministic, i.e., the specialized input always serves the same production, and slight alterations to the inputs require the same output Varied. Thus, to keep information stability, the cryptographic hash function is devoted to computing message hash values. Message integrity can be controlled by resolving the basic hash value, which is preserved with hash values joined by the same cryptographic hash function in the proposed theme.

One of the most popular systems for holding non-disapproval is a digital signature (Katz, 1996), a comparison of reviewing or guide. The seller types the information or the hash value of the message spread by a great hash function. It implements digital signals using asymmetric cryptography, which accepts a combination of civilized-private codes. To create clear there is no elimination, the shipper types the memorandum with the unique systems and the beneficiary controls the exporter's popular guide to accept the trademark. They determine it sets the unique key confidential; It is not computed-proper for a third team to solve a warning message without disqualifying the trademark. The issue that appears is that when someone uses the public sign to certify the essence of the sense, there is no state to provide certain that the popular key pairs that identification. For this purpose, an organized third team (it compels a certification authority to hold that a special public key applies to a unique body. They can receive digital signatures for security against non-discontent.

Although there are many parts of data integrity and non-disapproval, the development of blockchain infrastructure and a good contract technology frees up other aspects. Existing mechanisms for data integrity and non-elimination can be connected with blockchain features such as endurance, traceability and yield efficiency, and distribution functions.

\subsection{Blockchain Technology}

The application of blockchain technology that has been applied to the demand for biomedical data consumers (humans or similar programs) needs to take accurate and certified data from a biomedical database reference (Mytis-Gkometh, 2018).

Blockchain is a distributed transaction management technology that cannot be broken without a single party that can be trusted. The first Blockchain was proposed and implemented in Bitcoin (Nakamoto, 2008), a distributed infrastructure where users can carry out financial transactions with no regulators (e.g. banks). Other blockchain infrastructures are emerging, e.g. Authority (Buterin, 2014), where everyone can take part in the blockchain generation, and Hyper-ledger Fabric (Cachin, 2016), where only approved parties can post to the blockchain.

In the blockchain, it broadcasts each new transaction to a distributed network node; After all the nodes agree that the transaction is valid, it adds the transaction to the block. Each block contains a timestamp and a hash of the previous block and the transaction data, creating a chain that is unchanged and only additions. Copies of the entire blockchain are managed by each taking part node.

Some blockchain infrastructures, such as ETHEREUM1, support smart contracts, which are an irreversible computer code that runs on top of the blockchain. It can invoke the functions in the agreement in blockchain transactions.

Blockchain is a distributed database used to maintain a revolving record list called a block. Each block contains a timestamp and a link to the previous block. Blockchain is managed by a peer-to-path network to adhere to certain protocols to validate new blocks. (Cai, 2016), (Notheisen, 2017). This distributed ledger technology can be a framework that enables radical innovation in many areas. (Beck, 2017) Blockchain is a replicated distributed data structure and is shared among its network members. We introduced blockchain as a solution for double spending on Bitcoin. Because of how nodes on the Bitcoin network (called the miner) are validated and agree on the transactions that occur on the top of Bitcoin provides a container for an authoritative ledger transaction that determines who owns the TR Witnesses.

However, Blockchain can stand on its own with no need to relate to other parts. We can describe blockchain as a log whose record is in-batch with a block that is given a timestamp. It marks each block with a cryptographic hash. Each block refers to the previous block hash. This raises links between the blocks, thus creating a chain of blocks. Any Node connected to the list of blocks connected to the previous block (back-linked) can read and get an overview of the current state of the data being exchanged on the network. How blockchain works can be got by understanding how the Blockchain network works. This is a set of nodes (clients) that operate on the same blockchain through copies owned by each node.

A node can serve as an entry point for different users on the Blockchain, but for simplicity, we consider each user transacting on the Blockchain through their noses. (1) The user interacts with Blockchain through public and private key pair. They use private keys to sign their transactions, and we can trace their addresses through their public keys available on the network. Using asymmetrical cryptography carries integrity, authentication, and non-repudiation into the network. It broadcasts each signed transaction via a user node to a peer-to-no-jump. (2) A neighboring peer ensures that this transaction is valid before relay further. We will ignore invalid transactions. It distributes transactions across the network. (3) Transactions that have been collected and validated by the network using the above process within the agreed time range are sorted and packaged on a timestamp candidate that is given a timestamp. This process is called mining. Nome mining will redistribute this block into the network. (4) Other nodes will verify that the suggested block (a) contains valid transactions, and (b) refer to the previous block hash of the chain. In such cases, the block will be added to the chain. Otherwise, the block will be ignored. This marks the end of a cycle.

The way Blockchain works are seen in Figure 1. This process lasts. Blockchain is a set of contrasting authors who share databases without a trusted intermediary. To prevent the occurrence of chaos in this distributed environment, and to achieve a global consensus, every Network blockchain needs To carry out a set of rules that each database transaction must adhere to. I 
program this rule in each Blockchain client, which will then use the rules to check if a transaction is valid or not and, whether they will forward the transaction to the network or not.

Thus, Blockchain has a decentralized (1) feature; blockchain uses a distributed network model with the algorithm consensus to support data consistency in its network. (2) Persistent. It is almost impossible to cut or rollback transactions that have occurred in the Blockchain network. We can find blocks that contain invalid transactions at once. (3) Anonymous. Each user can interact in the Blockchain with the generated address, which does not reveal the user's real identity. This is a characteristic of Blockchain, although the anonymity of users cannot always be safeguarded because of its intrinsic limitations. (4) Can be audited, transactions within Blockchain can be verified and tracked. Bitcoin, for example, the use of Blockchain, stores user data on model Unspent Transaction Output (UTXO). Each transaction must refer to the previous operation that has not been used. We may divide blockchain into (a). Public, (lb). Private and (s). Consortium (Zheng, 2017).

Blockchain is one of the most disruptive technologies (Tapscott, 2016). Blockchain is a revolutionary technology in the $21 \mathrm{st}$ century. According to Swan, the blockchain has the potential to revamp a variety of social, economic, political, governmental and cultural life.

We should think about the Blockchain as another class of a thing like the Internet - a comprehensive information technology with tiered technical levels and multiple classes of applications for any form of asset registry, inventory, and exchange, including every area of finance, economics, and money; Hard Assets (physical property, homes, cars); and intangible assets (votes, ideas, reputation, intention, health data, information, etc.). But the Blockchain concept is even more; It is a new organizing paradigm for the discovery, valuation, and transfer of all quanta (discrete units) of anything, and potential for the coordination of all human activity at a much larger scale than has been possible before“. (Swan, 2015).

The existence of Blockchain technology has a long history and is associated with the group that called itself Cypherpunk. This group requires privacy in economic transactions and social connections in the open society and all electronics. When several parties make a transaction, they must ensure that each party only knows the content of the transaction. They must ensure the identity of the transaction party as small as possible revealed to the other party or the public.

"Privacy is necessary for an open society in the electronic age. Privacy is not secrecy. A private matter is something one doesn't want the entire world to know, but a secret matter is something one doesn't want anybody to know. Privacy is the power to reveal oneself to the world selectively ".

The emergence of its first blockchain is bitcoin to coincide with the global financial industry crisis in 2008, which causes the community to become unbelievers (distrust) of banking institutions. A person or group of people who named him Satoshi Nakamoto then published a paper titled "Bitcoin: A Peer-to-Peer Electronic Cash System" (Nakamoto, 2008). The paper provides a brief description of the protocol for transferring direct (peer-to-peer) electronic currencies using a cryptocurrency called Bitcoin.

At the beginning of its appearance, blockchain technology is defined with Bitcoin and Distributed Ledger Technology (DLT) which is the parent book (Ledger) that serves to record the various transactions that occur within the blockchain network. DLT itself is public, transparent and immutable, so they can audit it. But in subsequent developments, blockchain not only recorded transactions but began to enter the realm of economic, business, legal, political, humanitarian and scientific social. In the economic sphere, the most important innovations that Blockchain has to provide are payment transactions and decentralized exchanges, selling tokens, digital assets, and smart contracts. This innovation has not been in the era of network computer technology (web) before.

Other privileges of the blockchain are coordinating, recording, and transaction that has been done cannot be changed or canceled (tamper-proof). With the uniqueness of the architecture, blockchain can be a place to store various records of both individuals, organizations and communities in the form of documents, identities and digital assets. Besides being noted, ownership of the asset or property can be changed to the smart property by encrypting and providing a unique (digital signature) marker so that the asset can be tracked, controlled or exchanged within Blockchain. All assets that are tangible, such as a home or vehicle and various other assets that are intangible, can be registered and transacted in the blockchain. One of the digital assets that can be registered e.g. intellectual property rights (HAKI) such as patents and others.

Another major innovation of blockchain technology is the proving mechanism of a transaction that does not trust its users (trustless). They are quite entrusting to the bookkeeping system (ledger) which is public and interconnected through nodes that are decentralized and managed by miners (miners) acting as accountants. The miners have two functions as volunteers and accountants to help record transactions in the blockchain. The miner dedicates its computing resources to secure and process various transactions within the blockchain network. They compete against each other to verify the deal and then put it into a block that is arranged in chronological order. It connects the new block to the earlier block being secured using the "hash" act as a digital marker to make sure the authenticity of the transaction data. Every miner that enters a new block into the blockchain network will get a reward in the form of the digital coin (bitcoin), which can then a means of payment to buy goods and services or be stored as digital assets-Like Precious Metals (gold). The decentralized bookkeeping (database) is the antithesis of a centralized database that has been used. Although the centralized database has solved the double-spending issue, they must register all users on the central server to be operationalist. Thus, through a centralized database, it can be known to all users' identities along with their financial track record. Centralized databases are also an easy target to be hacked both inside and out because they have a central point of attack and failure. If a hacker can master the centralized database, then he or she can change the ownership of each available fund, steal from his or her legitimate owner or create a new token and then transfer the funds on his behalf.

By applying the blockchain, it can create verification of marketing without holding third-party help as negotiators. We can carry out selling between purchasers without taking to entrust database updates and ledger transactions to third parties. The Trust I attach it to the algorithm code that will prepare the operation when both parties have admitted. The Economist calls this technology a "trust machine." 
Blockchain itself continues to experience very much development. If on the blockchain 1.0 still related to Bitcoin as a digital currency and deposit instrument, the Blockchain 2.0 act has been expanding that can transfer assets and smart contracts and smart property. Blockchain 2.0 can register various assets both tangible and intangible. Tangible assets are assets in the much form, which are then coded into digital assets such as homeownership, hotel room rental, vehicle rental, ownership, or shared access to vehicles. The resources of the ethereal such as licenses, trademarks, copyrights and domain names on the Internet can also be registered and transferred through the blockchain. Here are a few classes and examples of the 2.0 Blockchain treatment development.

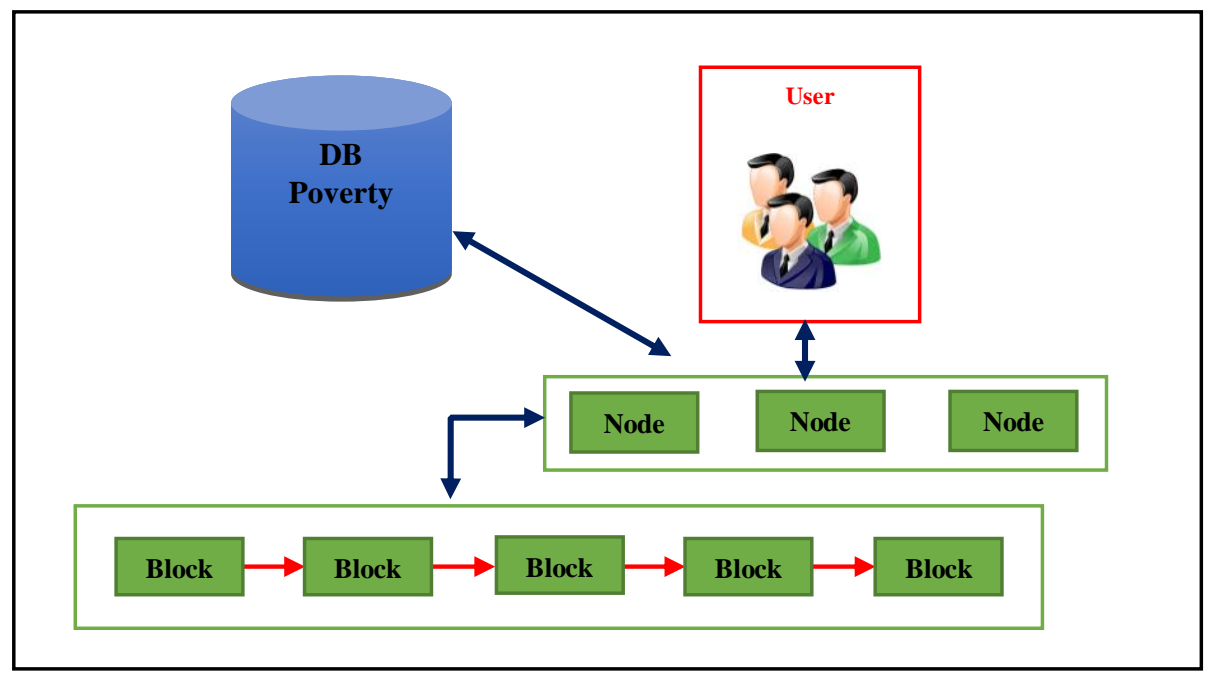

Fig 1: Poverty Data Retrieval Service Architecture

\section{RESEARCH METHOD}

They conducted a study using a literature review with a methodical examination (Dotsika, 2017). The literature related to the Blockchain is systematically examined for the need for an overview of the needs (requirement) required in the Poverty data retrieval service, particularly in relation to software, firmware and His taunted. Other literature related to Blockchain is also systematically examined to get an overview of the power of the Blockchain. After that, it conducts studies to recognize mechanisms that allow the mechanism to use Blockchain. In addition, there is also a search for use cases that can be used by using these mechanisms. Use-case is not restricted to a specific industrial realm but various industrial spheres. After the analysis with the model and use-case utilization of the Blockchain is made, it also analyzed the things that may inhibit its implementation. Ultimately, the results and the discussion will contain the model and use-case utilization.

\section{RESULT AND ANALYSIS}

Proceeds achieved from practice blockchain machinery in the handout of aid it makes to the resident regime in two schemes data calls (inquiring) and the legality evaluation of aid distribution messages as explained in Symbol 2 data Data applications and illustration 3 analyze conclusions.

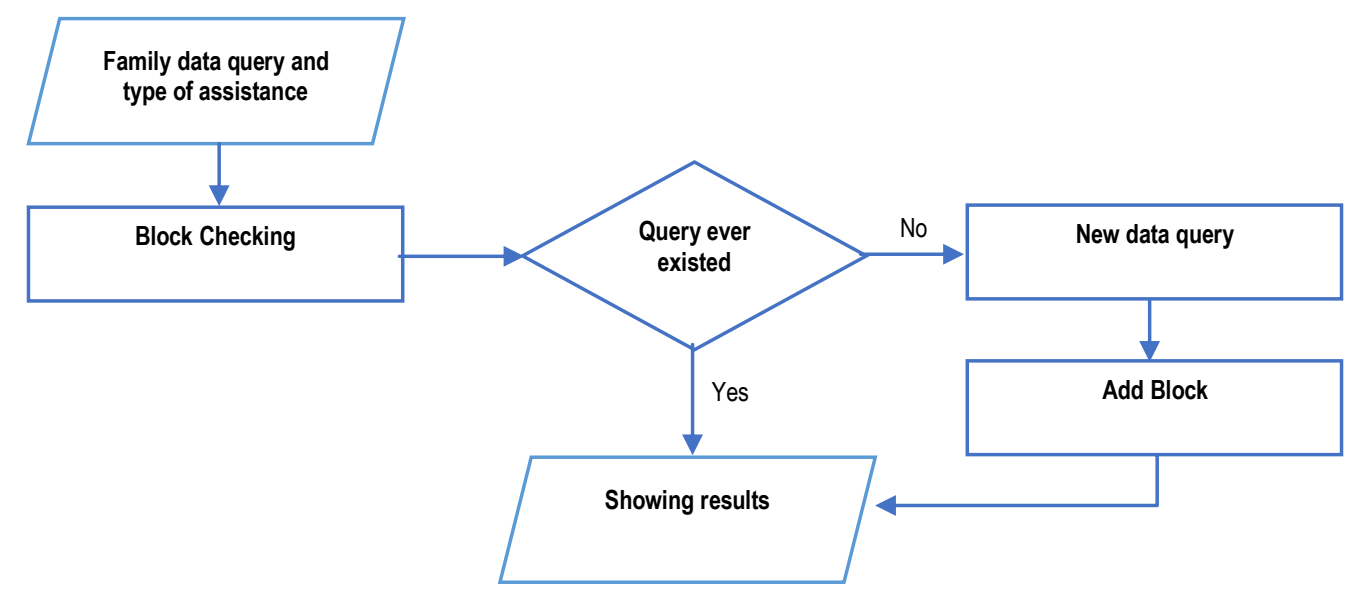

Fig 2: Data Request Algorithm 
Figure 2 shows a workflow service request to specify a family of type help to use blockchain technology, where the plot starts from the demand in the client application (node), the query results in the form of queries Poor family data and the help that has already been assisted. Further checking query results. The data request, if the query never existed, then it will form a new data block which is further added to the blockchain. It displays results either from the old block or from a new block to the user.

To find out if the harsh result of this query corresponds to the previous user request, then it is done by hash checking as shown in Figure 3 hash proof algorithm. Principle of hash proof with four circumstances:

A. Invalid or unproven hash

B. Invalid Hash with the existing condition of new results in the blockchain

C. Invalid Hash with no original result condition from the transaction database

D. Hash is valid with the status of no new result of the blockchain, but there is already a new result of the transaction database.

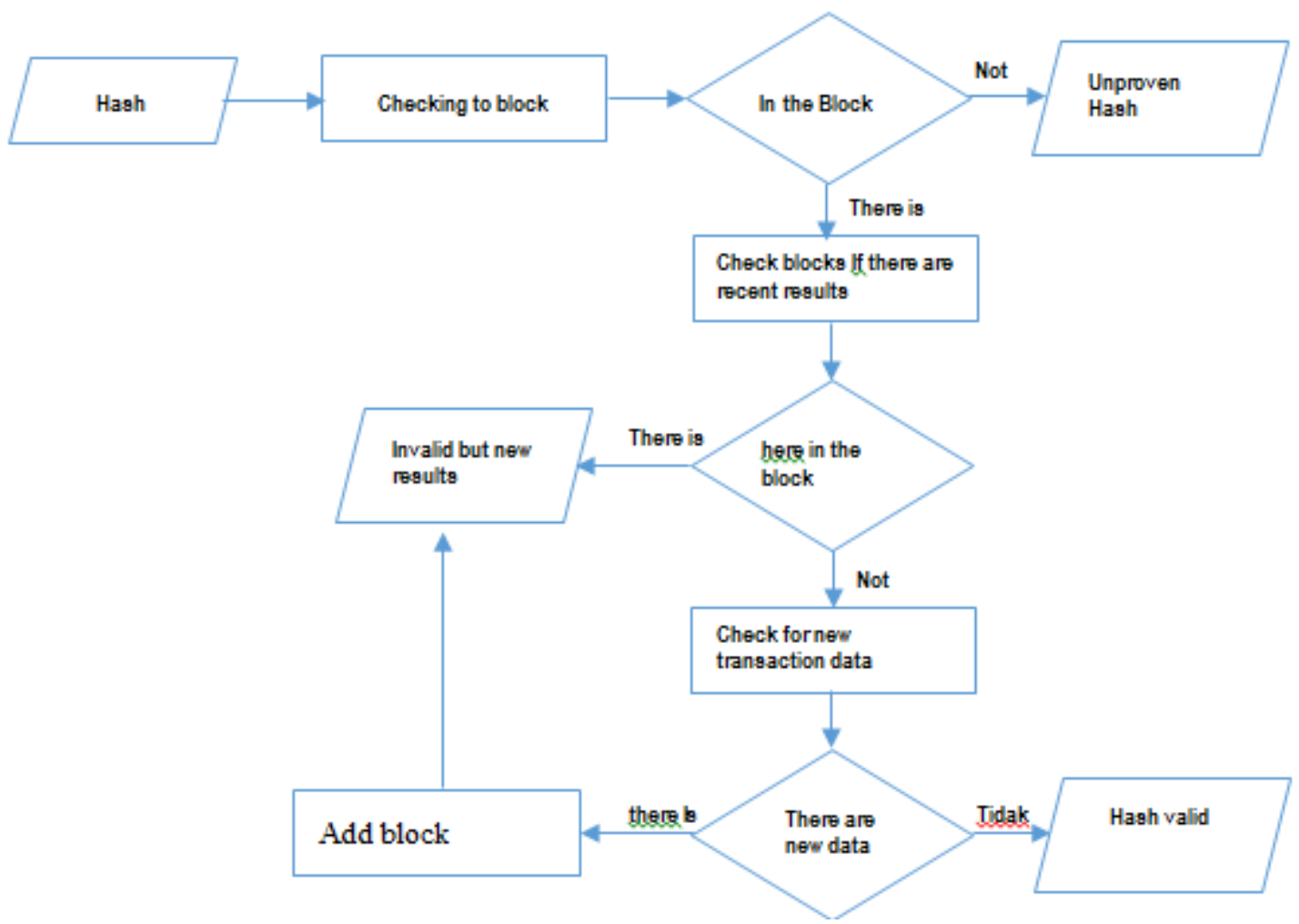

Fig. 3: Hash Proof algorithm

The Family data request interface and the help for cases (a) that have already received, (b) that have never received (data is in the blockchain) and (c) have never received help (Data is not in the blockchain, so it is requested from the database, Plus a new blockchain). As shown in the following figure: 4 :

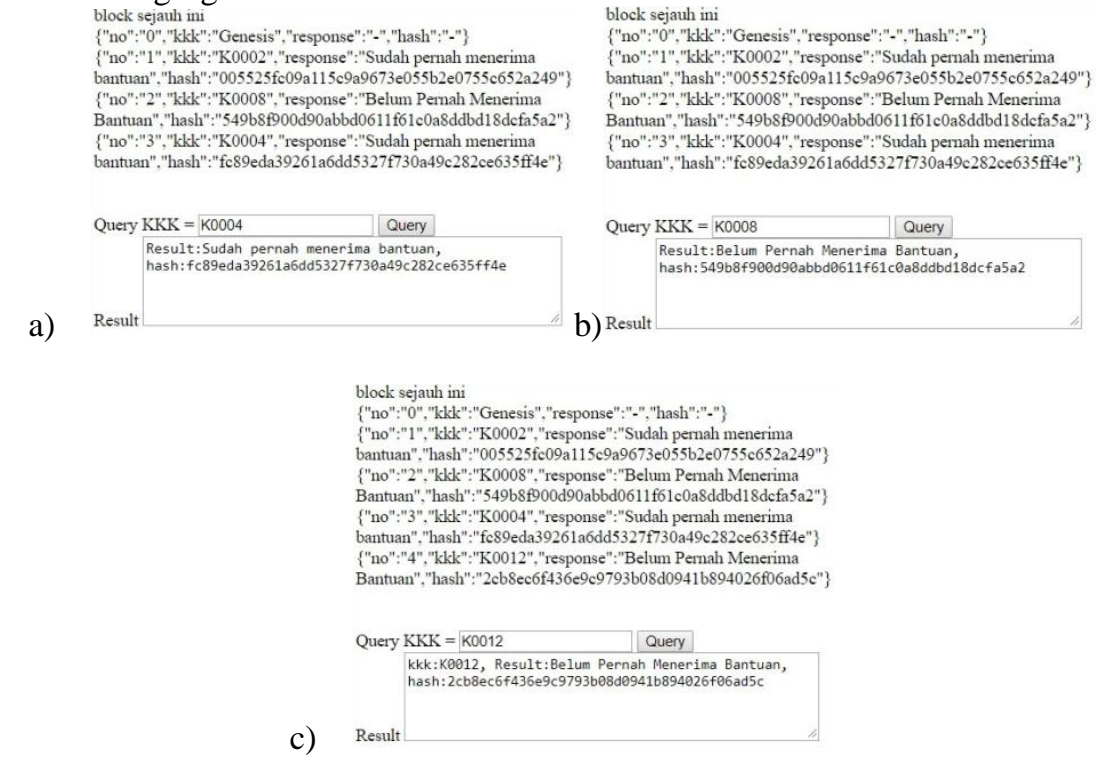

Fig 4: The interface queries data requests from Blockchain 
The result of the query by entering the code Family in the blockchain for the case (a) that has not and (b) that has received help in the blockchain got the query results in the form of family data with the status of receiving and yet received and the hash of which is Result of the Herblock in the blockchains. For cases (c) that have never received help (not in the blockchains) then, it sends the query to the database and includes in the new blockchain. Intermediate testing (validation) of the data hash of the recipient and the help type for a case (a) invalid hash, (s) a valid hash, with the condition, already present a new result on the blockchain, (c) correct hash, with the condition no new result of DB, (d) valid hash, with the state There is no new result of the blockchain but there is already a new result of DB.

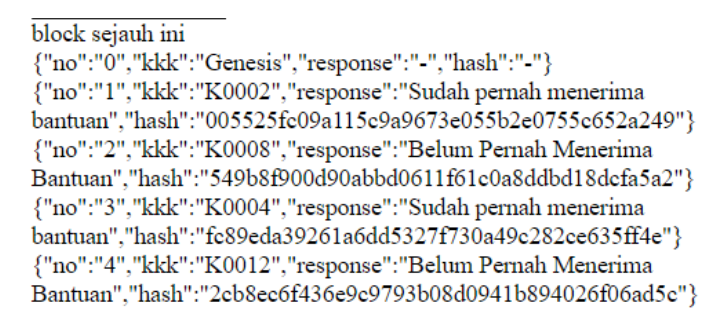

$\begin{array}{r}\text { Proof }=\text { inihashtidakvalid } \\ \text { Hash tidak valid }\end{array}$
a) Result block sejauh ini

$\{$ "no":"0","kkk":"Genesis","response":"-","hash":"-"\}

$\{$ "no":"1","kkk":"K0002","response":"Sudah pernah menerima

bantuan","hash":"005525fc09a115c9a9673e055b2e0755c652a249"\}

\{"no":"2","kkk":"K0008","response":"Belum Pernah Menerima

Bantuan","hash":"549b8f900d90abbd0611f61c0a8ddbd18dcfa5a2"\}

\{"no":"3","kkk":"K0004","response":"Sudah pernah menerima

bantuan","hash":"fc89eda39261a6dd5327f730a49c282ce635ff4e"\}

\{"no":"4","kkk":"K0012","response":"Belum Pernah Menerima

Bantuan","hash":"2cb8ec6f436e9c9793b08d0941b894026f06ad5c"\}

\{"no":"5","kkk":"K0008","response":"Sudah Pernah Menerima

Bantuan","hash":"e354551e 7745ec952c24173fa5cd23d5d6ca5bd0"

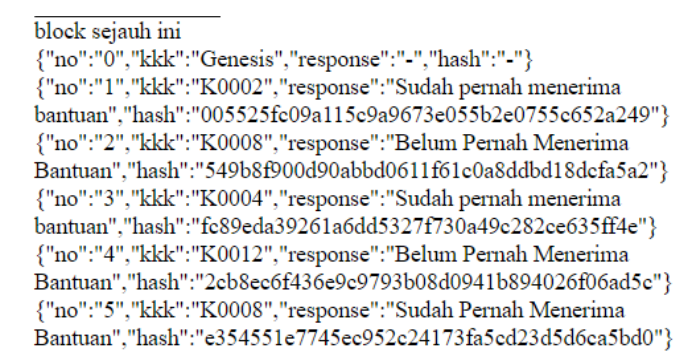

Proof $=005525 \mathrm{fc} 09 \mathrm{a} 115 \mathrm{c} 9 \mathrm{a} 9673 \mathrm{e} 055 \mathrm{~b} 2 \mathrm{e} 0755 \mathrm{c} 652 \mathrm{a} 249$ Valid dan tidak ada result baru dari db, status tetap sudah pernah menerima bantuan

c) Result
Validate

b) ${ }^{\mathrm{R}}$

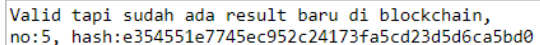

Proof $=2$ cb8ec6f436e9c9793b08d0941b894026f06ad5c

d) $)^{\operatorname{Re}}$

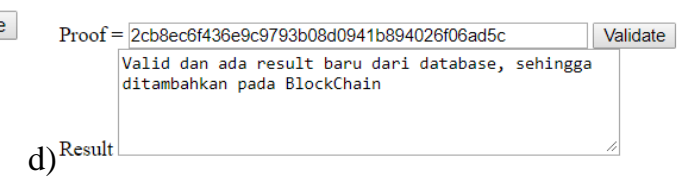

Fig. 5: Hash Testing interface

3. The result of the harsh test by entering a hash value in the blockchain, two possibilities are valid and invalid. For case (a) the hash value is tested if it does not match, then the result is an invalid or unsuitable hash value. For case (b) it tests the hash value if the result is a valid hash value with a condition already present the latest result on the blockchain. For case (c) The hash value is tested then the result is a valid hash value, with the condition no new result from DB, and for the case (d) The hash value is tested then the result is a valid hash value, with the condition no new result in the blockchain but already exists New result of DB.

\section{CONCLUSION}

Data retrieval services Blockchains Based on blockchain This article is one solution developed for the retrieval of poverty data in local governments hoping to minimize the manipulation of the help of data distribution in families Poor. The services developed in data retrieval using Blockchain method, where the process is done in the poverty database to request family data and type of help with checking on the blockchain for the status has received support and yet Receive help. For the existing data conditions in the blockchain, there are two statues that have received help and have received no help. As for data that does not exist in the blockchain, then it is requested in the database and added in the new blockchain. To test a hash value, there are two possibilities of the invalid or inappropriate hash value and a valid hash value. For valid conditions, there are three conditions i.e. a valid hash value with a condition of existing new result on the blockchain, with the condition of no new result of the database, and with the status of no new result of the blockchain but there is already a new result of the database. Data retrieval using the Blockchain method can solve the prevention of recipient data manipulation for aid distribution cases in a local government.

\section{REFERENCES}

Beck, R., \& Müller-Bloch, C. (2017). Blockchain as radical innovation: a framework for engaging with distributed ledgers as incumbent organization. 
Buterin, V. (2014). A next-generation smart contract and decentralized application platform. white paper, $3,37$.

Cachin, C. (2016, July). Architecture of the hyperledger blockchain fabric. In Workshop on distributed cryptocurrencies and consensus ledgers (Vol. 310, p. 4).

Cai, Y., \& Zhu, D. (2016). Fraud detections for online businesses: a perspective from blockchain technology. Financial Innovation, 2(1), 20.

Christidis, K., \& Devetsikiotis, M. (2016). Blockchains and smart contracts for the internet of things. Ieee Access, 4, $2292-2303$.

de Kruijff, J., \& Weigand, H. (2017, June). Understanding the blockchain using enterprise ontology. In International Conference on Advanced Information Systems Engineering (pp. 29-43). Springer, Cham.

Derbeko, P., Dolev, S., Gudes, E., \& Sharma, S. (2016). Security and privacy aspects in MapReduce on clouds: A survey. Computer science review, 20, 1-28.

Dotsika, F., \& Watkins, A. (2017). Identifying potentially disruptive trends by means of keyword network analysis. Technological Forecasting and Social Change, 119, 114-127.

Iankoulova, I., \& Daneva, M. (2012, May). Cloud computing security requirements: A systematic review. In 2012 Sixth International Conference on Research Challenges in Information Science (RCIS) (pp. 1-7). IEEE.

Katz, J., Menezes, A. J., Van Oorschot, P. C., \& Vanstone, S. A. (1996). Handbook of applied cryptography. CRC press.

Kleinaki, A. S., Mytis-Gkometh, P., Drosatos, G., Efraimidis, P. S., \& Kaldoudi, E. (2018). A blockchain-based notarization service for biomedical knowledge retrieval. Computational and structural biotechnology journal, 16, 288-297.

Mytis-Gkometh, P., Drosatos, G., Efraimidis, P. S., \& Kaldoudi, E. (2018). Notarization of knowledge retrieval from biomedical repositories using blockchain technology. In Precision Medicine Powered by pHealth and Connected Health (pp. 69-73). Springer, Singapore.

Nakamoto, S. (2008). Bitcoin: A peer-to-peer electronic cash system," http://bitcoin. org/bitcoin. pdf.

Notheisen, B., Hawlitschek, F., \& Weinhardt, C. (2017). Breaking down the blockchain hype-towards a blockchain market engineering approach.

Qu, F., Wu, Z., Wang, F. Y., \& Cho, W. (2015). A security and privacy review of VANETs. IEEE Transactions on Intelligent Transportation Systems, 16(6), 2985-2996.

Swan, M. (2015). Blockchain: Blueprint for a new economy. " O'Reilly Media, Inc.".

Tapscott, D., \& Tapscott, A. (2016). Blockchain revolution: how the technology behind bitcoin is changing money, business, and the world. Penguin.

Vigil, M., Buchmann, J., Cabarcas, D., Weinert, C., \& Wiesmaier, A. (2015). Integrity, authenticity, non-repudiation, and proof of existence for long-term archiving: a survey. Computers \& Security, 50, 16-32.

Zheng, Z., Xie, S., Dai, H., Chen, X., \& Wang, H. (2017, June). An overview of blockchain technology: Architecture, consensus, and future trends. In 2017 IEEE International Congress on Big Data (BigData Congress) (pp. 557-564). IEEE. 